\title{
Operación y Simulación de un Secador Spray para la Producción de Pentaborato de Sodio
}

\author{
Jorge W. Velasco ${ }^{(1,2,3)}$, Gloria del V. Villaflor ${ }^{(1,2,3)}$, Jorge E. Flores ${ }^{(1,2)}$ y Eduardo Bisonard ${ }^{(1,2)}$ \\ Universidad Nacional de Salta. (1) Instituto de Beneficio de Minerales. Facultad de Ingeniería, (2) Consejo \\ de Investigación, CIUNSa, (3) INIQUI. CONICET. Avenida Bolivia 5150 - 4400, Salta, Argentina \\ (e-mail: inbemi@unsa.edu.ar, villaflo@unsa.edu.ar, jeflores@unsa.edu.ar)
}

Recibido Ene. 11, 2014; Aceptado Mar. 18, 2014; Versión final recibida May. 15, 2014

\begin{abstract}
Resumen
Se realiza el estudio experimental de las condiciones de operación de un secadero spray para la obtención de pentaborato de sodio. Se determina la cantidad de agua a evaporar, la temperatura de la solución a alimentar, y las temperaturas de entrada y salida de los gases de combustión que van al secadero spray. Se realizan experiencias para seleccionar el tipo de boquilla y la presión a utilizar, a fin de lograr una buena pulverización, que favorezca la evaporación de la solución y formación de cristales. Se analizan distintas alternativas para minimizar el agua de disolución, los consumos energéticos y las pérdidas de calor en el sistema. Para simular el proceso, se desarrolla un programa de computación que realiza los balances de materia y energía, determinando las variables de interés. Se concluye que el programa desarrollado es adecuado y permite obtener información rápida y confiable sobre diversas variables del proceso.
\end{abstract}

Palabras clave: simulación, secador spray, pentaborato de sodio, balance de materia, balance de energía

\section{Simulation and Operation of a Spray Dryer for the Production of Sodium Pentaborate}

\begin{abstract}
An experimental study of the operating conditions of a spray dryer for obtaining sodium pentaborate is presented. The amount of water evaporated the temperature of the feeding solution and inlet and outlet temperatures of the combustion gases going to the spray dryer are determined. Experiments are performed for selecting the type of nozzle and the pressure to be used to achieve good spraying that favors the evaporation of the solution and the formation of crystals. Various options to minimize the amount of dilution water, the energy consumption and the heat losses in the system are analyzed. To simulate the process, a computer program that performs the energy and material balances and determines the variables of interest is developed. It is concluded that the computer program is an adequate tool for obtaining values of the several variables of the process in a reliable and fast way.
\end{abstract}




\section{INTRODUCCIÓN}

El pentaborato de sodio, $\mathrm{NaB}_{5} \mathrm{O}_{8} .5 \mathrm{H}_{2} \mathrm{O}$ o $\mathrm{Na}_{2} \mathrm{O}_{1} .5 \mathrm{~B}_{2} \mathrm{O}_{3} .10 \mathrm{H}_{2} \mathrm{O}$, es un borato de alto valor agregado. Es una sal alcalina que se comercializa en forma de fino polvo blanco, soluble en agua dando soluciones con alto contenido de boro (Stella Mary, 2008). Es estable en contacto con su solución saturada entre 2 y $59,5 \stackrel{\circ}{ } \mathrm{C}$. Por debajo de $2{ }^{\circ} \mathrm{C}$ forma bórax y por arriba de $59,5{ }^{\circ} \mathrm{C}$ el borato de Taylor $\left(2 \mathrm{Na}_{2} \mathrm{O} .9 \mathrm{~B}_{2} \mathrm{O}_{3} .11 \mathrm{H}_{2} \mathrm{O}\right.$, p.e.1,90). El pentaborato de sodio tiene una amplia gama de aplicaciones, siendo la más común en la industria de los fertilizantes (Alarcón Vera, 2001), en aplicaciones foliares por boro deficiencia, como regulador del crecimiento vegetal. Se usa como pesticida (Ware y Whitacre, 2004), tiene propiedades fungicidas, protege contra los hongos, las cucarachas, los grillos, las moscas y los milpiés. Es desfoliante, elimina las hojas anchas de las plantas, por lo que es comúnmente utilizado en la producción de algodón para quitar las hojas antes de la cosecha. Es retardador de llamas (Wilkie y Morgan, 2009), aislante e inhibidor de la corrosión (Flores et al, 2012). Se usa además como anticongelante, fundente de soldadura y en el tratamiento de maderas, resguardándola del ataque de microbios y termitas (Lebow, 2004).

El pentaborato de sodio puede obtenerse por calentamiento de una mezcla estequiométrica de bórax decahidratado y ácido bórico en un secador rotativo o en un secador por pulverización (spray), alimentando una solución de composición apropiada, siendo más efectivo el segundo proceso. En este caso, la mezcla de bórax y ácido bórico se disuelve en agua en un tanque agitado calefaccionado y el producto obtenido se evapora en un secador spray, permitiendo un íntimo contacto entre fases y logrando una buena calidad en el producto obtenido (Perry, 2000; Nonhebel, 2002). La reacción de obtención de pentaborato de sodio es:

$\mathrm{Na}_{2} \mathrm{~B}_{4} \mathrm{O}_{7} .10 \mathrm{H}_{2} \mathrm{O}+6 \mathrm{H}_{3} \mathrm{BO}_{3} \quad \longrightarrow \quad 2 \mathrm{NaB}_{5} \mathrm{O}_{8} .5 \mathrm{H}_{2} \mathrm{O}+9 \mathrm{H}_{2} \mathrm{O}$

Otro método consiste en obtener el pentaborato por cristalización por enfriamiento a partir de una solución caliente de composición apropiada. Alternativamente se puede mezclar ácido bórico y bórax granular, iniciándose la reacción con un pequeño agregado de agua y continuando la misma por efecto del agua liberada por la reacción (Flores, 2004). Asimismo se han propuesto otros métodos que emplean ulexita, con el agregado fraccionado de ácido sulfúrico obteniendo pentaborato de sodio como producto intermedio (Domínguez et al, 2011) o preparando una pulpa en agua de alta ley, burbujeando anhídrido carbónico (Alkan et al, 1991) o anhídrido sulfuroso (Alkan et al, 1987). La elección del método de obtención de pentaborato de sodio resultará de un análisis comparativo de los costos de producción involucrados (Flores y Tinte, 2008).

Los secadores por pulverización son de gran importancia en numerosas operaciones de secado industrial. El estudio de esta tecnología permite determinar las variables operativas que minimicen la contaminación ambiental producida por los efluentes gaseosos y maximicen el ahorro energético del proceso, fundamentalmente por disminución del agua a evaporar en la etapa de secado. Un aspecto de operabilidad muy importante es la acumulación de gotas/partículas en las paredes del secador y en las regiones de salida; el método de Lagrange (Langrish y Fletcher, 2003; Fletcher et al, 2006) permite determinar cuándo llegan a las paredes las gotitas individuales.

\section{TRABAJO EXPERIMENTAL}

Las materias primas utilizadas en el presente trabajo para la obtención de la solución de pentaborato de sodio son ácido bórico y bórax decahidratado técnico granular. La temperatura de la solución de alimentación al equipo se fija en el rango de 50 a $70 \stackrel{\circ}{\circ}$, establecido en función de datos experimentales obtenidos previamente. En base a esto se prepara la solución teniendo en cuenta las cantidades de agua mínima a utilizar, que en la etapa posterior de secado debe ser eliminada.

Las experiencias a escala piloto, se realizan utilizando un secador spray diseñado y construido en la planta piloto del Instituto de Beneficio de Minerales (INBEMI), de la Facultad de Ingeniería de la Universidad Nacional de Salta. El secador es cilíndrico-cónico, con la parte cilíndrica de $3 \mathrm{~m}$ de altura y $2 \mathrm{~m}$ de diámetro y la parte cónica de $1,8 \mathrm{~m}$ de alto con una salida de 0,30 m. El equipo está construido totalmente de acero inoxidable con compuerta inferior para la extracción del producto. Los conductos, desde el quemador al secadero y desde éste al ciclón, son de acero inoxidable de sección rectangular con clapetas que permiten hacer circular los gases en cocorriente o en contracorriente indistintamente. La salida del spray se conecta a un ciclón para separar los finos arrastrados. Se emplean pulverizadores con boquillas intercambiables para suministrar diferentes tipos de niebla. Se utiliza un quemador monotobera presurizado de $85000 \mathrm{kcal} / \mathrm{h}$ alimentado con gas natural. Se colocan termocuplas para medir las temperaturas de los gases a la entrada y salida del secadero. En la figura 1 se muestra un esquema del secadero spray utilizado. 


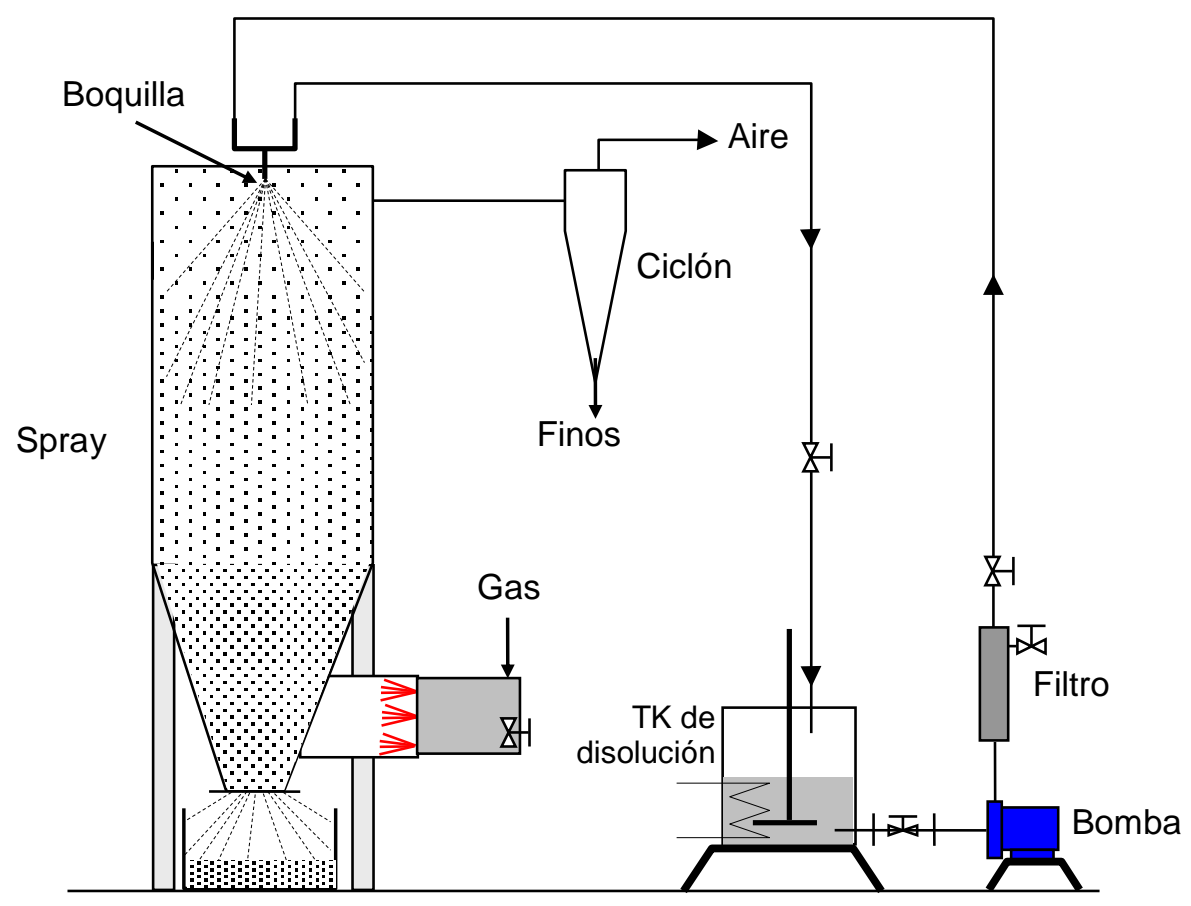

Fig. 1. Diagrama simplificado del proceso de obtención de pentaborato de sodio.

Se realizaron experiencias previas para seleccionar el tipo de boquilla y la presión de trabajo en las mismas, a fin de lograr una buena pulverización que favorezca la evaporación de la solución y la formación y crecimiento de los cristales. El ángulo de diseminación de las boquillas debe ser tal que el choque contra la pared ocurra a una distancia aproximada de cuatro veces el diámetro de la cámara, teniendo el cono de descarga una pendiente de por lo menos 60ำ (Perry, 2000).

Los ensayos experimentales se efectuaron utilizando una boquilla estática de plástico resistente con orificios pequeños a fin de disminuir el tamaño de las gotas formadas y mejorar la pulverización, midiéndose los caudales a diferentes presiones en la boquilla, entre 10 y $20 \mathrm{lb} / / \mathrm{in}^{2}$. Asimismo se realizaron ensayos con boquillas metálicas con turbinas, trabajando con presiones de hasta $40 \mathrm{lb}_{f} / \mathrm{in}^{2}$. A presiones bajas las boquillas producen gotas grandes y la solución no es correctamente pulverizada, describiendo un cono muy pequeño. A mayores presiones el cono descripto por la solución pulverizada es el correcto reduciéndose la formación de gotas grandes. Las boquillas con turbina, que dieron mejores resultados, fueron seleccionadas para las experiencias que se llevan a cabo actualmente.

La solución de pentaborato, contenida en el tanque de disolución a la temperatura de trabajo, se alimenta con una bomba centrífuga al pulverizador del secador spray, previo pasaje por un filtro que retiene impurezas sólidas arrastradas. Los ensayos se realizaron con la entrada de los gases de combustión en contracorriente, ingresando por la base del cilindro y saliendo por la parte superior del mismo. La niebla formada en el pulverizador se pone en íntimo contacto con los gases calientes ascendentes y se forman copos de pentaborato de sodio, los que caen, descargándose por la parte cónica del secadero. Se miden, entre otros, los caudales de alimentación, la velocidad de de los gases, los consumos de gas natural y las temperaturas a la entrada y a la salida del spray con termocuplas posicionadas próximas al quemador, al ciclón y al pulverizador.

Los análisis químicos de los cristales obtenidos tanto a la salida de sólidos del spray como en el ciclón dan un contenido promedio de $\mathrm{B}_{2} \mathrm{O}_{3}$ del $57,5 \%$, siendo el valor teórico para el pentaborato de sodio puro y seco de $58,98 \%$.

\section{PROGRAMA DE CÁLCULO}

Se realiza un programa de cálculo en Excel para efectuar los balances de materia y de energía para la obtención de pentaborato de sodio. Para una dada producción de pentaborato, el programa establece las cantidades estequiométricas de reactivos a procesar. Teniendo en cuenta los datos de solubilidades de ácido bórico, bórax decahidratado y pentaborato de sodio, se obtiene la cantidad de agua que se debe agregar, a diferentes temperaturas, para solubilizar el bórax decahidratado y el ácido bórico y verificar que el pentaborato de sodio obtenido se encuentre en solución a la temperatura de trabajo. 
Se calcula la cantidad de agua producida por la reacción de obtención de pentaborato (ecuación 1) y a partir de la misma y de la cantidad de agua obtenida con las solubilidades, se obtiene la cantidad de agua mínima a agregar al proceso. Esta es la cantidad de agua que debe ser evaporada en la etapa posterior en el secadero spray. También se analizan experimentalmente distintas alternativas para minimizar el agua de disolución, que debe ser eliminada en la etapa de secado, a fin de obtener un ahorro de la energía consumida, con la consecuente disminución del $\mathrm{CO}_{2}$ emitido, causante del cambio climático.

El programa permite trabajar con distintos tipos de combustible, gas natural, GLP o fueloil para calcular la energía térmica necesaria para el proceso de secado proveniente de la combustión del combustible seleccionado. Conocida la composición del combustible a utilizar el programa calcula el poder calorífico del mismo, el caudal y la composición de los gases de combustión producidos (Villaflor et al, 2006). Se calculan además el calor necesario para evaporar el agua de la solución y, a partir de éste, el calor a liberar por el combustible en base a la eficiencia térmica del proceso. El calor liberado por el combustible depende del flujo y del poder calorífico inferior del mismo.

\section{RESULTADOS}

Se realizan los cálculos para producir $100 \mathrm{~kg}$ de pentaborato de sodio utilizando gas natural como combustible, tomando para el mismo una composición típica correspondiente a un gas natural del norte argentino. Se calculan la cantidad y la composición de los gases de combustión producidos para determinados excesos de aire seco o húmedo.

En la figura 2 se muestran, a modo de ejemplo, algunos resultados obtenidos con el programa, tales como cantidades de calor a entregar y liberar para evaporar el agua, flujo de gas natural necesario y de gases de combustión producidos, para obtener $100 \mathrm{~kg}$ de pentaborato de sodio a distintas temperaturas del proceso y eficiencias térmicas.

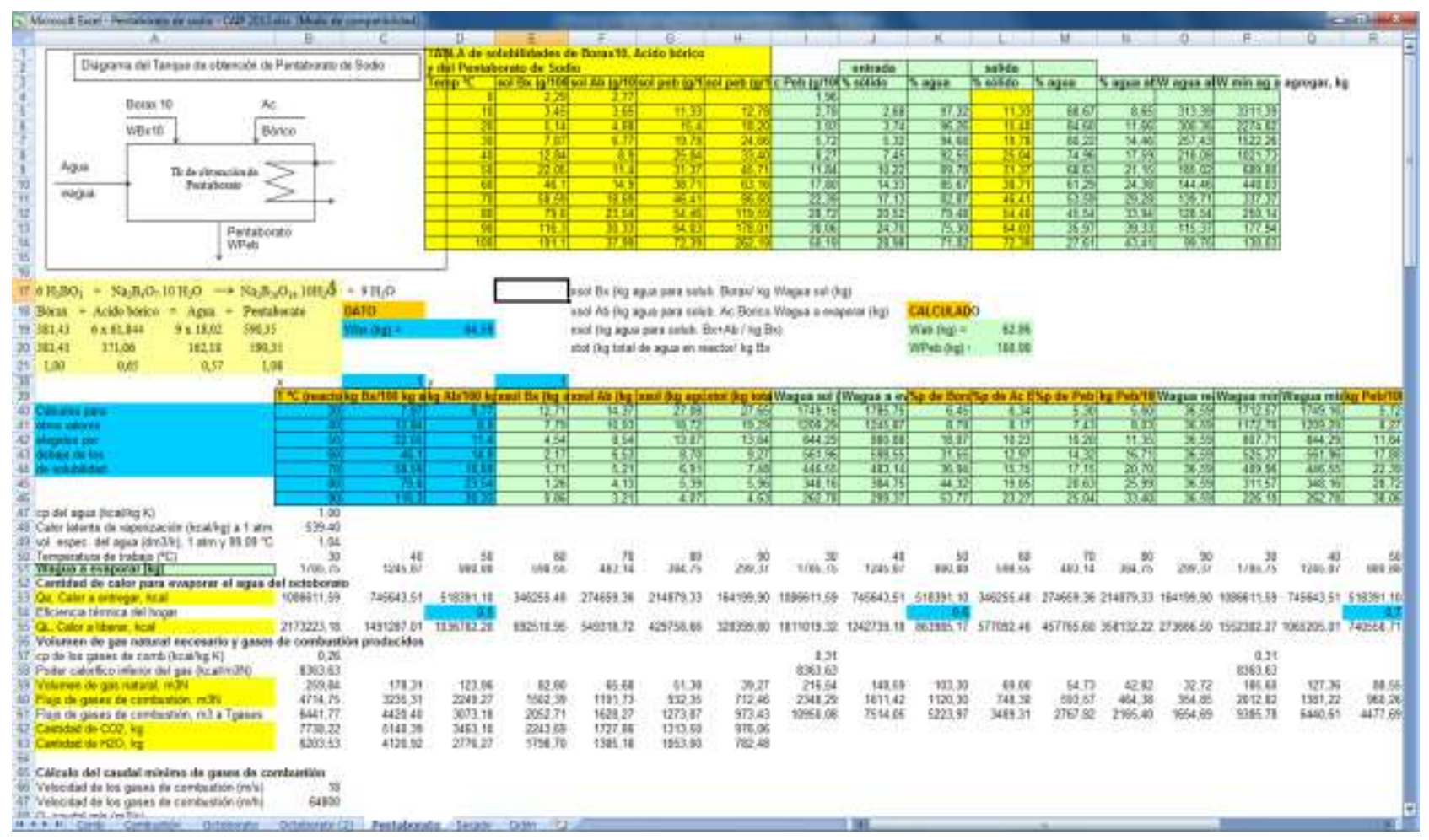

Fig. 2. Resultados obtenidos con el programa para diferentes temperaturas de operación.

En la figura 3 se muestra la variación de la cantidad de agua economizada en función de la temperatura de operación. En la figura 4 se grafican tanto los calores a entregar, $Q_{e}$, y liberar, QL, como los flujos de gas natural utilizado, $F_{\mathrm{GN}}$, y de gases de combustión producidos, $\mathrm{F}_{\mathrm{g}}$, en función de la temperatura de operación, para una eficiencia térmica del sistema fijada. De acuerdo a las temperaturas de trabajo fijadas, entre 50 y $70 \stackrel{\circ}{\circ}$, se obtiene un ahorro de agua del 20 al $30 \%$, siendo el mismo significativo y consecuentemente disminuyen, en el orden del 50 al $75 \%$ aproximadamente, el consumo de combustible, los gases de combustión generados y los calores involucrados, para una eficiencia térmica del $60 \%$. En la figura 5 se muestra, a modo de ejemplo, los resultados del programa para un ensayo experimental realizado en planta piloto con el secadero spray ya descripto. 


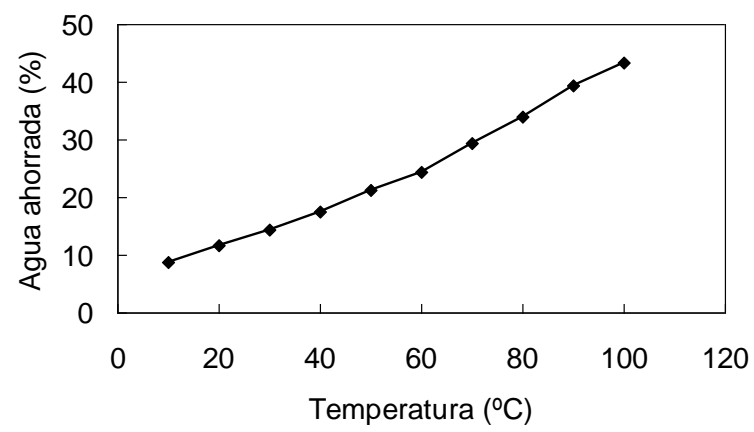

Fig. 3. Porcentaje de agua ahorrada en función de la temperatura de operación.

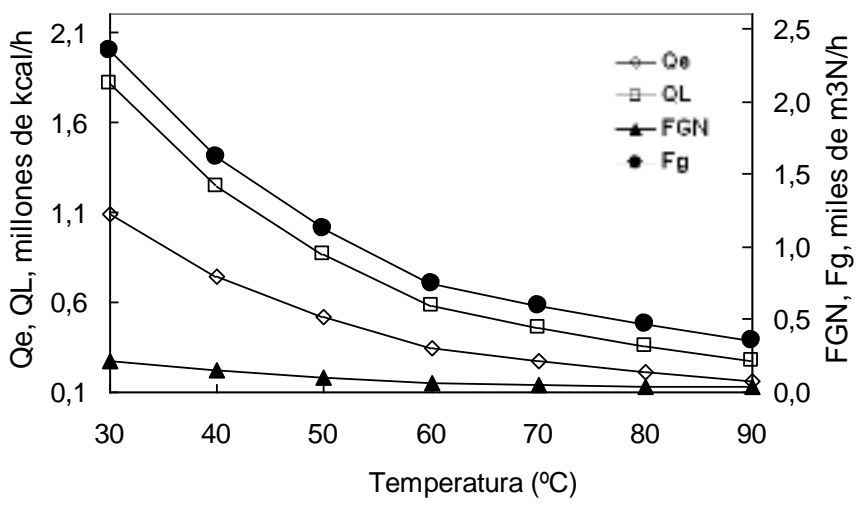

Fig. 4. Calores a entregar y liberar y flujos de gas natural y gases de combustión producidos.

En esta planilla se cargan las mediciones realizadas en los ensayos experimentales, tales como las temperaturas a la entrada y salida del equipo, la velocidad de los gases a la salida del ciclón, el volumen de gas natural suministrado al quemador y los volúmenes de solución alimentada. Se obtienen como resultados el caudal de gas natural consumido, el caudal de la solución procesada, la cantidad de agua evaporada, los calores involucrados y las cantidades de sólido descargado, tanto en la cámara spray como en el ciclón. Estos resultados verifican los obtenidos por el programa de simulación, en especial la cantidad de pentaborato de sodio producida, siendo dichos valores concordantes si se tiene en cuenta los errores experimentales y las pérdidas en el proceso (cañerías, bomba, juntas, aislamiento, manipulación, etc.) que se producen en todo trabajo experimental a nivel de planta piloto. La disminución de estas pérdidas en el proceso experimental aumentará la eficiencia total del proceso, disminuyendo asimismo los costos de producción.

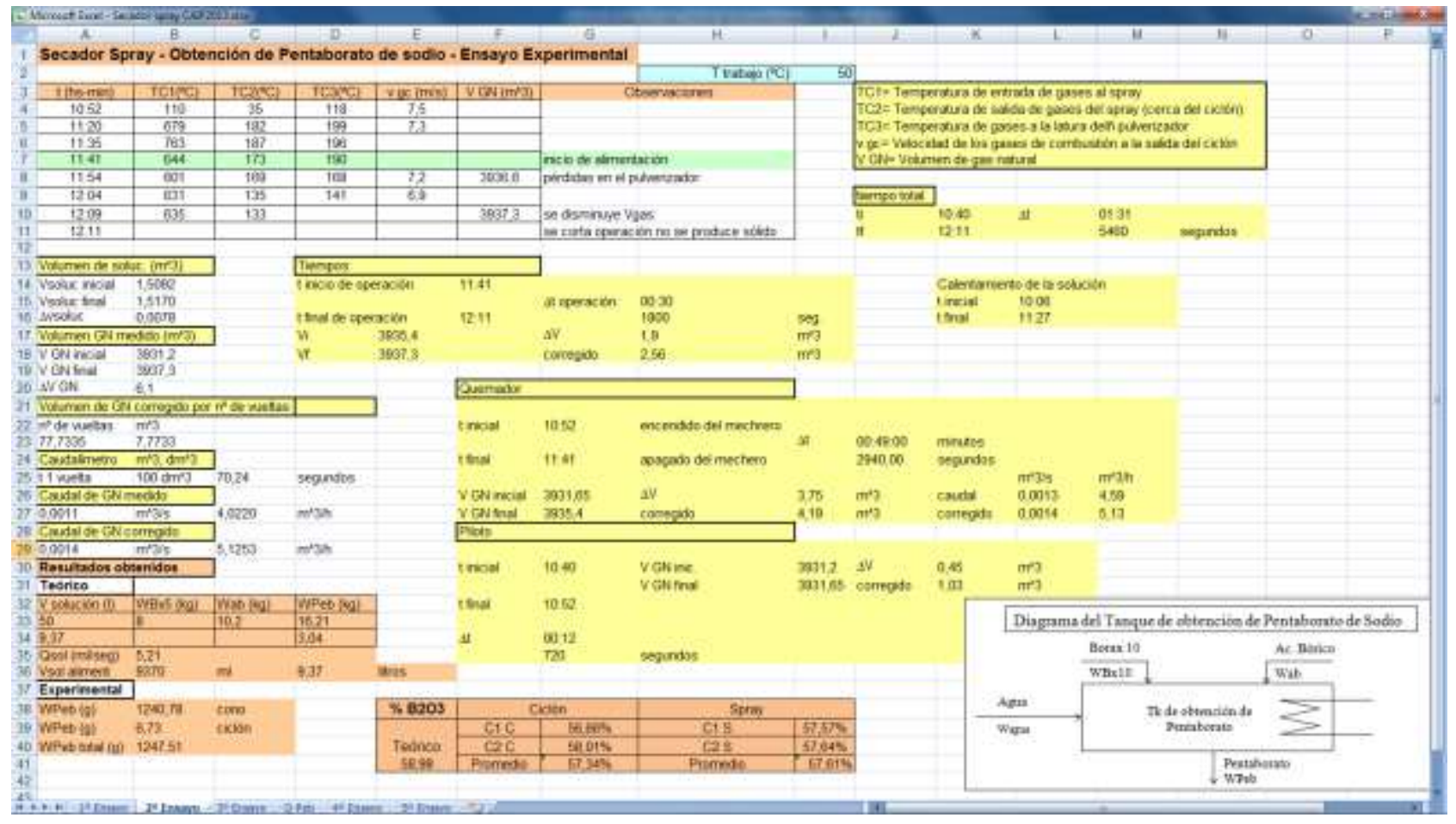

Fig. 5. Resultados del programa para un ensayo experimental a $T_{\text {sol }}=50 \stackrel{\circ}{C}$

\section{CONCLUSIONES}

Los trabajos experimentales realizados permiten obtener las siguientes conclusiones: i) El control de la temperatura de la solución de pentaborato a atomizar debe ser riguroso a fin de evitar la precipitación por enfriamiento. ii) Es fundamental el control de las temperaturas de entrada y salida de los gases de calefacción, siendo recomendable comenzar la alimentación de la solución cuando la temperatura de salida es de $200 \stackrel{\circ}{\circ}$ aproximadamente. iii) Las boquillas con turbina dieron mejores resultados que las estáticas 
proporcionando menor tamaño de las gotas formadas. iv) Los análisis químicos de los cristales de pentaborato de sodio obtenidos dan un contenido promedio de $\mathrm{B}_{2} \mathrm{O}_{3}$ del $57,5 \%$, siendo el valor teórico de $58,98 \%$.

Por otra parte, el programa desarrollado permite realizar los balances de materia y energía del proceso de obtención de pentaborato de sodio, logrando información rápida y confiable del mismo, permitiendo analizar: i) La cantidad necesaria de agua de disolución, así como distintas alternativas para minimizarla, a fin de obtener un ahorro de la energía consumida en la evaporación. ii) Los consumos energéticos del proceso y las pérdidas de calor en el sistema. iii) Las emisiones de gases producidas y su disminución como consecuencia del ahorro del agua de disolución.

\section{REFERENCIAS}

Alarcón Vera, A. L. Horticultura. XIX(6), 36-46 (2001).

Alkan M. y M.M. Kocakerim. Dissolution kinetics of ulexite in water saturated by sulphur dioxide. J. Chem. Tech. Biotechnol., 40, 215-222 (1987).

Alkan M., M. Oktay, M.M. Kocakerim y Z. Karagolge. Dissolution kinetics of borate minerals in $\mathrm{CO}_{2}$ saturated water. Hydrometallurgy, 26, 255-262 (1991).

Domínguez O., E. Serrano, J. Flores y R. Michel. Alternativa para Mejorar el Proceso de Producción de Acido Bórico. Información Tecnológica. 22(1), 101-108 (2011).

Fletcher, D.F., B. Guo, D.J.E. Harvie, T.A.G. Langrish, J.J. Nijdam y J. Williams. What is important in the simulation of spray dryer performance and how do current CFD models perform? Applied Mathematical Modelling, 30, 1281-1292, (2006).

Flores, H. R. El beneficio de los boratos. Crisol Ediciones, Salta, Argentina (2004).

Flores, H. R. y M. A. Tinte. Cálculo del costo comparativo de la obtención de concentrados de boratos. Información Tecnológica. 19(3), 3-6 (2008).

Flores, H. R., G. Villaflor y M. A. Tinte Montalbetti. Penta y octoborato de sodio. Ed. EUNSa, Salta, Argentina (2012).

Langrish, T. A. G. y D. F. Fletcher. Prospects for the Modelling and Design of Spray Dryers in the 21st Century. Drying Technology. Vol. 21(2), 197-215, (2003).

Lebow S., J. Winandy y D. Bender, Treated Wood in Transition: A Look at CCA and the Candidates to Replace It. Design Focus. 14(2), 3-8 (2004).

Nonhebel, G., A. A. H. Moss, El secado de sólidos en la industria química. Ed. Reverté, Barcelona, España (2002).

Perry, R., D. Green y J. Maloney, Perry Manual del Ingeniero Químico. Mc Graw Hill, V, D. F., México (2000).

Stella Mary, S., S. S. Kirupavathy, P. Mythili, y R. Gopalakrishnan. Growth and characterization of sodium pentaborate $\left[\mathrm{Na}\left(\mathrm{H}_{4} \mathrm{~B}_{5} \mathrm{O}_{10}\right)\right]$ single crystals. Spectrochimica Acta Part A, 71, 1311-1316 (2008).

Villaflor, G., G. Morales y J. Velasco. Variables Significativas del Proceso de Combustión del Gas Natural. Información Tecnológica. Vol 19(4), 57-62, Chile (2008).

Ware, G. \& D. Whitacre, The Pesticide Book, Meister Publication, 6th ed., Ohio, USA (2004).

Wilkie C. A. y A. B. Morgan. Ed., Fire Retardancy of Polymeric Materials, CRC Press, Taylor and Francis Group (2009). 